\title{
Subtipos de trastorno específico del desarrollo del lenguaje: perfiles clínicos en una muestra hispanohablante
}

\author{
N. Crespo-Eguílaz, J. Narbona
}

\author{
SUBTYPES OF SPECIFIC LANGUAGE IMPAIRMENT IN SPANISH-SPEAKING \\ CHILDREN: A CLUSTER ANALYSIS OF LINGUISTIC FEATURES
}

\begin{abstract}
Summary. Introduction. Specific language impairment (SLI) can be viewed as a continuum with different subtypes depending on the combination of deficits and strengths in each of the components of linguistic behavior. Aim. To analyze the phenomenological profiles in a correlative series of Spanish children in order to facilitate their endophenotypic differentiation and the choice of strategies of intervention. Patients and methods. 86 referred children, aged 4 to 9 years, with a mean non-verbal inteligence quotient of 93 (range 72-114) and accomplishing clinical criteria of SLI. Apart from clinical interview and neurological examination, a protocol of questionnaires and tests was used in all subjects in order to measure their formal (phonological, syntactic, lexical) and functional (semantic and pragmatic) linguistic abilities. A cluster analysis of variables was used in order to investigate SLI subtypes. Results. In the total sample, a $24 \%$ of whole series have a pure phonological expressive disorder. 55\% of subjects have mixed receptivelexpressive disorders; from these, the most pervasive subtype is due to verbal agnosia (11\%), but more frequently observed subtypes affect syntax reception and expression combined to difficulties in phonological programming (36\%) or in lexical retrieval (8\%). Furthermore, $21 \%$ of the total sample present with difficulties in pragmatic use of language even if their formal linguistic abilities and non-verbal behavior are normal. Conclusions. Our empyrical approach confirms that the admitted SLI subtypes in international literature applies to Spanish-speaking children for endophenotyping and intervention-planning purposes. [REV NEUROL 2006; 43 (Supl 1): S193-200]

Key words. Anomic-syntaxic disorder. Cluster analysis. Phonological disorder. Phonological-syntaxic disorder. Pragmatic deficit disorder. Spanish speaking children. Specific language impairment. Verbal auditory agnosia.
\end{abstract}

\section{INTRODUCCIÓN}

El trastorno específico del lenguaje (TEL) o disfasia es una dificultad innata, duradera y relativamente autónoma para la adquisición y manejo del código lingüístico. Se señala que es una dificultad innata porque se manifiesta desde el inicio del desarrollo lingüístico del sujeto, que surge con retraso y lo hace con determinadas distorsiones que diferencian al TEL del retraso simple del lenguaje, y se diferencia de los trastornos adquiridos, incluso en época temprana, tras un período de desarrollo lingüístico normal. En la definición del TEL también se señala que es un trastorno duradero porque, o bien acompaña al sujeto de forma manifiesta a lo largo de su vida, o bien, en muchos casos de menor gravedad -tras una relativa 'normalización' de los recursos lingüísticos formales con el paso de los años y con la ayuda de las intervenciones logopédicas-, la afectación innata del lenguaje puede manifestarse ulteriormente e interferir en el proceso de aprendizaje de la lectoescritura, en la construcción del discurso verbal complejo (descripción, narración, conversación), y en las modalidades de trabajo mental que se apoyan en el lenguaje (inferencias, cálculo, etc.). Por último, en la definición se destaca que el TEL es una afectación relativamente autónoma (específica) del desarrollo del lenguaje, ya que no depende de un déficit sensorial, motriz, intelectual o socioemocional que lo justifique;

\section{Aceptado: 14.09 .06}

Departamento de Pediatría. Unidad de Neurología Pediátrica. Clínica Universitaria. Universidad de Navarra. Pamplona, Navarra, España.

Correspondencia: Dra. Nerea Crespo Eguílaz. Departamento de Pediatría. Clínica Universitaria. Universidad de Navarra. Avda. Pío XII, s/n. E-31080 Pamplona (Navarra).E-mail: necrespo@unav.es

Trabajo parcialmente subvencionado por la Fundación Fuentes Dutor a la Unidad de Investigación en Neurología del Desarrollo de la Clínica Universitaria de Navarra.

(C) 2006, REVISTA DE NEUROLOGÍA es un trastorno intrínseco del procesamiento del lenguaje. Esto último es precisamente lo que ha dado pie al calificativo de trastorno específico y a las definiciones por exclusión que se encuentran en la bibliografía [1-3]. Al matizar que su autonomía es relativa también se hace referencia al hecho de que el TEL puede coexistir con retraso intelectual, con trastornos de la gama autista, etc. [4,5], y que es preciso tener en cuenta las influencias recíprocas entre lo estrictamente lingüístico y el resto de las variables cognitivas y comunicativas.

Algunos niños con disfasia tienen dificultad en el procesamiento de índices temporales del orden de algunas decenas de milisegundos [6] y en la discriminación entre estímulos auditivos cuando su diferencia de duración es muy corta o cuando se dan en una sucesión rápida, pero esta dificultad desaparece cuando los estímulos se presentan de forma ralentizada [7]. La velocidad de procesamiento en niños disfásicos en actividades lingüísticas y no lingüísticas es generalmente más lenta que la de niños con un lenguaje normal [8,9]. Recientemente se está poniendo énfasis en el hecho de que los niños con TEL pueden presentar dificultades en la memoria verbal a corto plazo (memoria de trabajo fonológica), y esto hace que tengan problemas para retener las representaciones de las formas fonológicas [10-15]. La repetición de pseudopalabras, como medida de memoria fonológica de trabajo, suele considerarse como un buen marcador psicolingüístico en relación con la identificación de esta patología [16-23]. La percepción temporal y la memoria de trabajo verbal son imprescindibles para la conciencia fonológica y ésta es importante también para la descodificación grafofonémica de la lectura; por lo tanto, algunos niños disfásicos -de forma especial los que presentan trastornos fonológicos- tienen un riesgo alto de desarrollar trastornos de lectura, y esto influye en los distintos dominios académicos [20,24-30].

Las investigaciones actuales por grupos de genética molecular muestran un significativo ligamiento en diferentes regiones del 
genoma (13q21, 16q24 y 19q13) y los datos apoyan el modelo poligénico multifactorial [31-34]. La variada sintomatología y la heterogeneidad que caracteriza al TEL hace patente la necesidad de hacer subgrupos, no sólo para precisar la descripción del fenotipo con vistas a los estudios geneticomoleculares, sino además para facilitar una identificación e intervención tempranas [35].

La fenomenología clínica del TEL puede revestir diversas formas según la proporción en que se vean afectados cada uno de los aspectos formales o funcionales del lenguaje; ello supone unos perfiles específicos de deficiencias y capacidades con pronóstico y respuesta a la intervención diferentes [36]; aunque todos ellos se agrupen en una sola categoría nosológica por su aparición desde el inicio del desarrollo, por la aparente ausencia de lesiones neurológicas y de carencias socioambientales y sensoriales y por la posible existencia de un continuo entre las posibles formas $[1,37,38]$.

La clasificación universalmente conocida de Rapin y Allen [39-42] se basa en el análisis fenomenológico de series amplias de niños con TEL. En la citada clasificación se distinguen los subgrupos siguientes: trastorno fonológico, dispraxia verbal, trastorno fonológico-sintáctico, agnosia verbal, trastorno léxico-sintáctico y trastorno semántico-pragmático. Otras clasificaciones del TEL con base empírica proceden de estudios con medidas psicolinguiísticas como los de Aram y Nation [43] y Conti-Ramsden et al $[44,45]$; estos últimos aplicaron una serie de tests de lenguaje a niños disfásicos, realizaron análisis estadísticos muy sólidos y determinaron seis subtipos que se aproximan a los propuestos por Rapin y Allen.

Dentro de la gama autista $[46,47]$ se pueden encontrar todas las variantes de trastornos lingüísticos que se describen en el TEL, excepto las puramente expresivas [48-50]: los niños autistas situados en los niveles de mayor gravedad del espectro presentan alteraciones graves en todos los componentes del lenguaje, mientras que los autistas de nivel alto o con síndrome de Asperger se caracterizan por dificultades en los aspectos semántico y pragmático del lenguaje [49]. Sin embargo, se diferencian de los niños con TEL porque presentan además alteraciones sociales, del pensamiento y de la conducta [51,52], y no utilizan estrategias comunicativas de carácter no verbal para compensar las dificultades linguísticas. Algunos autores consideran el autismo, el síndrome de Asperger y el trastorno semántico-pragmático como cuadros solapados que forman parte de un continuo [53,54]. Si se emplean los criterios nosotáxicos del DSM-IV TR [55] y del CIE-10 [56], se puede considerar la ubicación del déficit pragmático, o semántico-pragmático, en la categoría de trastornos generalizados del desarrollo sin otra especificación. En los estudios actuales se considera la pragmática como un aspecto más de la competencia lingüística; así que puede haber déficit lingüísticos que afecten especialmente a la estructura del lenguaje, a su uso funcional o a ambos [57,58], sin afectación de las otras habilidades intersubjetivas y de relación social o de la conducta, aunque también hay que tener en cuenta que los niños con TEL con déficit que afecta sólo a las dimensiones formales del lenguaje pueden presentar -como consecuencia de dicho déficit- dificultades en la competencia social y en el uso de estrategias comunicativas y una participación menor en las interacciones, en comparación con los niños con un desarrollo del lenguaje normal [25,59-62]. Por lo tanto, dentro de los programas de intervención, es importante incorporar desde el principio pautas de uso del lenguaje en un contexto comunicativo incluso cuando el objetivo inmediato sea la mejora de los aspectos formales [61,63-65].
En la actualidad se pretende establecer la diferenciación de los subtipos de TEL en función de las áreas de procesamiento lingüístico afectadas, es decir, ateniéndose a los procesos psicológicos de comprensión y/o expresión o a las dimensiones lingüísticas de fonología, morfología, sintaxis, semántica y pragmática $[58,66]$. La mayor parte de las investigaciones -si no todas- que aportan distintas tipologías de TEL utilizan muestras de sujetos disfásicos de habla inglesa. Con nuestro estudio pretendemos ofrecer una evidencia empírica acerca de los subtipos de TEL en una muestra de niños afectos, españoles hispanohablantes. Consideramos que la importancia de una clasificación diagnóstica radica en la identificación de la sintomatología concreta del niño para orientar la intervención psicopedagógica y logopédica más adecuada y para afinar en la descripción de fenotipos de TEL que permita mejorar los resultados de los estudios genéticos.

\section{PACIENTES Y MÉTODOS}

Nuestro estudio ha utilizado una muestra formada por 86 sujetos (61 niños y 25 niñas) con TEL. Estos pacientes han sido reclutados de forma correlativa en la consulta de la Unidad de Neurología Pediátrica de la Clínica Universitaria de Navarra. El diagnóstico clínico del TEL (basado en los datos anamnésicos y en la apreciación de que el niño presenta una anomalía del lenguaje discrepante del resto de las funciones cognitivas) se complementa con los resultados de la evaluación neuropsicolingüística realizada posteriormente. Las edades de los sujetos están comprendidas entre 3,5 y 9,5 años; los 6 años son la media.

La media del cociente intelectual (CI) no verbal del grupo es 93, a partir de la escala manipulativa internacional de Leiter $[67,68]$ o con la subescala manipulativa de la escala Wechsler de inteligencia revisada (WISC-R) [69]. El 9\% tiene un CI > 115; en el 73\% de la serie el CI está comprendido entre 85-114 y el 18\% tiene un CI limítrofe. Las habilidades lingüísticas de cada sujeto son bajas respecto al resto del desarrollo cognitivo.

\section{Evaluación psicolingüística}

Con las evaluaciones psicolingüísticas -realizadas todas ellas por los autores del trabajo- se ha cuantificado el rendimiento de cada sujeto en las dimensiones formales y funcionales (que constituyen ocho variables psicolingüísticas) mediante las siguientes pruebas:

\section{Evaluación de los componentes formales en la vertiente receptiva}

- Discriminación fonológica. Subtest de gnosias auditivofonéticas de la serie de exploración del lenguaje para preescolares (BELP-P) [70] e integración auditiva del test de Illinois de aptitudes pscicolingüísticas (ITPA) [71].

- Comprensión de vocabulario. Test de vocabulario en imágenes Peabody [72].

- Comprensión de sintaxis. Comprensión auditiva del ITPA [71] y la escala de comprensión del test de sintaxis de Aguado (TSA) [73].

Evaluación de los componentes formales en la vertiente expresiva

- Programación fonológica. Praxias bucofaciales del BELP [70], articulación de palabras y logotomas del test de Spreen y Benton [74] adaptado al castellano por Mendilaharsu et al [75] y registro fonológico inducido [76].

- Denominación. Test de vocabulario de Boston [77].

- Sintaxis expresiva. Integración gramatical del ITPA [71] y la escala de expresión del TSA [73].

\section{Evaluación de los componentes funcionales}

- Uso cognitivo. Definición de conceptos -vocabulario de la escala McCarthy de aptitudes y psicomotricidad (MSCA) [78]-; acceso al léxico a partir de diversas categorías de conceptos (fluencia verbal de la MSCA [78]); cohesión discursiva del relato -memoria de historias del test de memoria y aprendizaje (TOMAL) [79]-; categorización (semejanzas de la WISC-R [69]) y definiciones (vocabulario de la WISC-R [69]).

- Uso pragmático. Children's Communication Checklist (CCC) [80,81], versión castellana para su uso en esta investigación. 


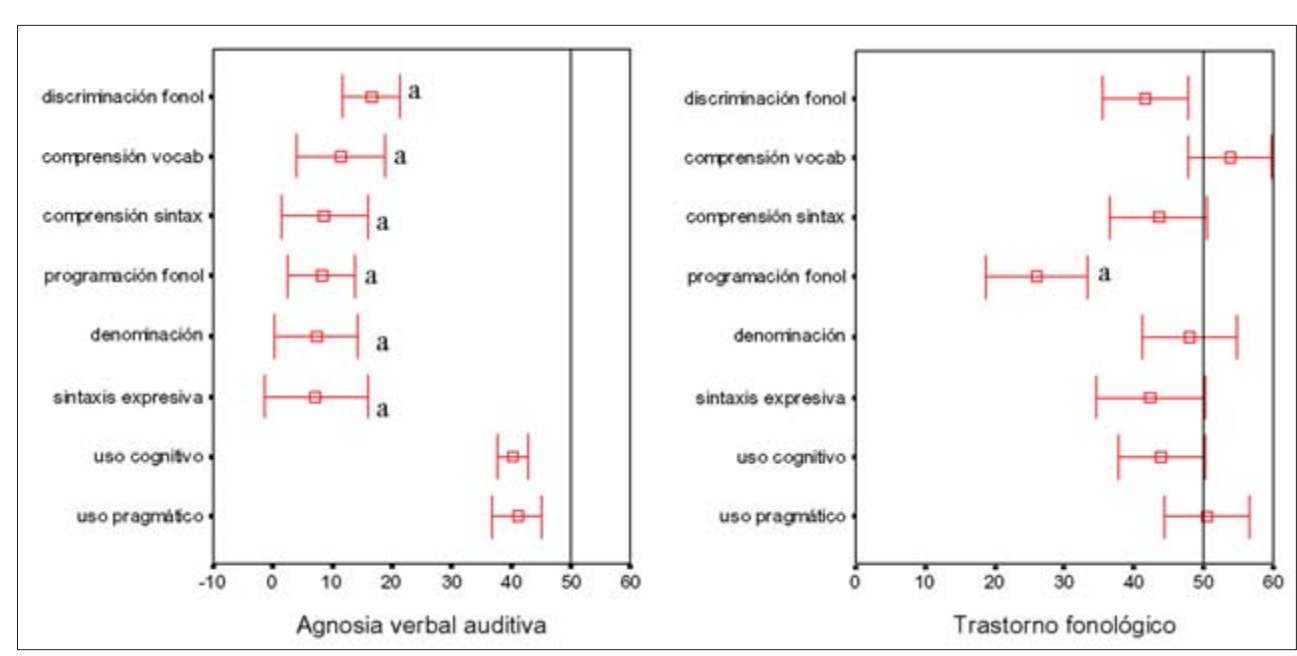

Figura 1. Subgrupos del trastorno específico del lenguaje (TEL) extremos en cuanto al grado de gravedad: con afectación masiva en las vertientes de comprensión y expresión (agnosia verbal auditiva) y con afectación de la vertiente expresiva (trastorno fonológico). Diferencia significativa con el resto de los subgrupos con TEL. Puntuaciones tipificadas T (media: 50; desviación típica: 10). ${ }^{a} p<0,01$.

Tabla I. Subtipos del trastorno específico del lenguaje de la casuística estudiada.

Afectación de la forma del lenguaje en

las vertientes de comprensión y expresión

Trastorno masivo: agnosia verbal auditiva

Trastorno fonológico-sintáctico

Trastorno léxico-sintáctico

Afectación de la forma del lenguaje en la vertiente expresiva

Trastorno fonológico

Afectación de las funciones psicolingüísticas

Trastorno pragmático

Trastorno semántico-pragmático

En cursiva se especifica la denominación que adoptamos para los distintos trastornos, acorde con las principales clasificaciones hoy en uso.

\section{Clasificación de los sujetos: análisis de conglomerados}

La evaluación psicolingüística nos permite valorar el nivel de desarrollo psicolingüístico alcanzado, detectar las dificultades específicas y determinar el grado de desviación correspondiente. Elaboramos un perfil de la conducta psicolingüística de cada niño examinado; éste resulta útil para conseguir una apreciación de conjunto de los puntos débiles y fuertes de cada sujeto y para organizar los datos en la investigación de un grupo con la misma patología.

Con la técnica estadística multivariante 'análisis de conglomerados' (cluster analysis) clasificamos a los participantes de nuestra casuística en grupos homogéneos, no conocidos de antemano, basados en las similitudes entre sus componentes a través de la correlación múltiple entre las distintas variables.

\section{Perfiles clínicos}

Cada conglomerado obtenido (a partir de aquí nos referiremos a ellos como subtipos de TEL) se corresponde con un perfil clínico que contempla las ocho variables lingüísticas estudiadas. Aportamos los datos descriptivos (puntuaciones medias y desviaciones estándares) y una valoración cualitativa de la sintomatología específica de cada grupo.

En cada una de estas variables medimos -con un análisis de varianza (ANOVA)- la significación estadística entre las medias de todos los subtipos de TEL. De esta forma pretendemos señalar los criterios significativos de clasificación, teniendo en cuenta el perfil específico y la gravedad.
RESULTADOS

Subtipos clínicos del TEL y perfiles psicolingüísticos

El TEL constituye una categoría clínica global que afecta a distintos dominios lingǘsticos de forma variable, con unos perfiles psicolingüísticos específicos. En la tabla I se indican los subtipos del TEL de nuestra casuística y, a continuación, se presentan los perfiles psicolingüísticos de cada uno de ellos.

Afectación de la forma del lenguaje en las vertientes de comprensión y expresión

Dentro de los trastornos mixtos en las vertientes de comprensión y expresión, se distingue la afectación masiva derivada de una agnosia verbal congénita y otros dos trastornos menos graves que combinan dificultad en la sintaxis receptiva y expresiva con trastorno de la fonología (trastorno fonológico-sintáctico) o de acceso al léxico (trastorno léxicosintáctico).

Agnosia verbal auditiva (Fig. 1). Es la afectación masiva del lenguaje desde el nivel más básico de reconocimiento de los diferenciadores fonéticos. La incapacidad para reconocer y discriminar los sonidos del lenguaje es el déficit básico de este grupo. La comprensión verbal es muy baja (menor de -3 desviaciones típicas por debajo de la media); los niños afectos comprenden palabras sueltas con el apoyo de lectura labial. Éstos se comportan funcionalmente como sordos para el lenguaje, aunque su audición es normal. Como el trastorno de la integración perceptiva afecta al desarrollo del resto de los componentes receptivos (sintaxis y vocabulario) y el de la expresión verbal, ésta también es nula o muy escasa y no pueden repetir palabras adecuadamente. Este subtipo se corresponde con el subtipo 'agnosia auditivoverbal' de la clasificación de Rapin y Allen. Los afectados comprenden y comunican las demandas e intereses mediante gestos naturales y, cuando utilizan el escaso lenguaje oral que llegan a adquirir, éste es adecuado para el contexto comunicativo. La buena capacidad de comunicación gestual y la normal empatía nos permiten diferenciar a los niños con agnosia verbal de los sujetos con trastorno de la gama autista (si bien en el espectro autista la agnosia verbal auditiva es frecuente). En cinco casos de la muestra se ha observado una mejoría al utilizar en la intervención ayudas a través de la lectura, es decir, al acceder a lo verbal por la vía visual, eludiendo o apoyando con la grafía la descodificación auditiva fonológica. La agnosia verbal auditiva es el TEL de mayor gravedad (Fig. 1); se diferencia estadísticamente de todos los subtipos en todas las variables formales del lenguaje $(p<0,01)$.

Los otros dos subtipos con afectación mixta (trastornos fonológico-sintáctico y léxico-sintáctico) tienen en común un déficit en sintaxis expresiva que los diferencia del grupo con déficit fonológico y de los trastornos de las funciones psicolingüísticas $(p<0,05)$. En ambos el discurso es simplificado y con errores sin patrón fijo, lo que afecta al uso de los afijos gramaticales (flexiones verbales, concordancias de género y número), de las palabras sintácticas (preposiciones, conjunciones, adverbios, artículos) y del orden mismo de los elementos de la frase. A su vez, estos dos subtipos de TEL se diferencian entre sí principalmente en la programación fonológica, que es significativamente menor en el primer grupo $(p<0,01)$, y en el vocabulario, que es significativamente menor en el segundo $(p<0,05)$.

Trastorno fonológico-sintáctico (Fig. 2). Los niños de nuestra casuística agrupados aquí tienen dificultades de comprensión cuando el enunciado incluye estructuras sintácticas complejas, es largo o se presenta descontextualizado. La fonología está alterada en la vertiente expresiva; los errores fonológicos (omisiones, distorsiones y sustituciones) afectan a la inteligibilidad. El discurso es hipofluente e hipogramatical. El nivel de vocabulario expresivo es reducido en el $42 \%$ de los sujetos de este grupo, aspecto no 
señalado en la bibliografía [3]. No se evidencian dificultades en las dimensiones funcionales del lenguaje.

Según lo indicado, los déficit de este grupo se centran en la fonología expresiva y, sobre todo, en la sintaxis receptiva y expresiva (más acusadas en esta última). El perfil es similar al subtipo con 'trastorno fonológico-sintáctico' de la taxonomía de Rapin y Allen.

Dentro de este subtipo con déficit fonológico y sintáctico incluimos a seis pacientes cuyas características se asemejan a la 'dispraxia verbal' descrita en las fuentes bibliográficas anglosajonas [39-41,82,83]: la emisión verbal es escasa, hablan con mucho esfuerzo y con poca fluidez e inteligibilidad y presentan dificultades para la realización de gestos bucales no verbales. Sin embargo, constatamos que presentan además un déficit en la comprensión y expresión de la sintaxis, por eso los clasificaciones dentro del trastorno mixto fonológico-sintáctico.

Trastorno léxico-sintáctico (Fig. 2). Los sujetos de la muestra que forman este grupo presentan un nivel pobre de comprensión de vocabulario. Estos problemas léxicos limitan la comprensión de enunciados. Esto puede deberse en parte a que no utilizan el bootstrapping sintáctico; es decir, que no deducen parte del significado de las palabras nuevas a partir de las características sintácticas de la frase [84-86]. No obstante, el déficit principal es la evocación lexical; esta habilidad es significativamente menor $(p<0,05)$ que en los demás subtipos con TEL (excepto en la agnosia verbal). Les cuesta encontrar la palabra correcta; por consiguiente, muestran anomias, pobreza terminológica, circunloquios y autocorrecciones frecuentes. Estas dificultades influyen en el uso semántico del lenguaje para relacionar significados, para mantener el referente a lo largo de las proposiciones y para expresar una idea. Como grupo, no presentan dificultades en la contextualización del lenguaje. Este subtipo se asemeja al 'déficit léxico-sintáctico' descrito por Rapin y Allen.

\section{Afectación de la forma del lenguaje en la vertiente expresiva}

Trastorno fonológico (Fig. 1). Se diferencia significativamente del resto de los grupos $(p<0,01)$-excepto de la agnosia verbal- en la variable 'programación fonológica'; las dificultades en esta dimensión lingüística son el problema único de este grupo, que muestra una capacidad media en la vertiente receptiva del lenguaje. Los sujetos de este grupo presentan un habla sin particulares problemas de fluidez, pero caracterizada por la imprecisión articulatoria y por los cambiantes defectos de pronunciación. Pueden producir fonemas y sílabas aisladamente, pero éstos se desestructuran u omiten de manera diversa al utilizarlos en el contexto de la palabra y/o frase. El nivel de vocabulario expresivo es aceptable, aunque con errores en la pronunciación en todos los sujetos, y el discurso es parcialmente ininteligible. Se aprecia una mejoría articulatoria en tareas de repetición, respecto a la de emisión espontánea. No presentan dificultades en el uso cognitivo y pragmático del lenguaje. Las características psicolingüísticas de este subtipo se corresponden con el grupo con 'trastorno de la programación fonológica' de Rapin y Allen.

\section{Afectación de las funciones psicolingüísticas}

Se diferencian de los demás grupos con TEL en el uso funcional del lenguaje en la comunicación $(p<0,01)$, mientras que las dimensiones formales del lenguaje están aceptablemente preservadas (Fig. 3).

Trastorno pragmático. En este grupo no se aprecian problemas en los aspectos formales del lenguaje (fonología y morfosintaxis) ni en las referencias semánticas de las emisiones verbales. Sin embargo, los afectados presentan un déficit específico en la adaptación del lenguaje al contexto y al interlocutor: el discurso comunicativo tiende a centrarse en sus temas de interés, realizan una elevada cantidad de inicios de conversación, interrupciones y cambios inesperados del tema de conversación. En una situación controlada pueden realizar definiciones, descripciones y relatos coherentes. Los sujetos incluidos en este subtipo muestran afectación específica del uso pragmático del lenguaje, pero no tienen dificultades significativas en ninguna otra dimensión lingüística.

Trastorno semántico-pragmático. Los sujetos componentes de este grupo muestran un trastorno pragmático, pero además un déficit en el uso cogniti- 
Tabla II. Frecuencia de la muestra por subtipos y sexo.

\begin{tabular}{lcccc}
\hline & $n$ & Frecuencia & Niños & Niñas \\
\hline Agnosia verbal & 9 & $11 \%$ & 6 & 3 \\
\hline Trastorno fonológico-sintáctico & 31 & $36 \%$ & 19 & 12 \\
\hline Trastorno léxico-sintáctico & 7 & $8 \%$ & 7 & 0 \\
\hline Trastorno fonológico & 21 & $24 \%$ & 17 & 4 \\
\hline Trastorno pragmático & 7 & $8 \%$ & 5 & 2 \\
\hline Trastorno semántico-pragmático & 11 & $13 \%$ & 7 & 4 \\
\hline Total & 86 & $100 \%$ & 61 & 25 \\
\hline
\end{tabular}

vo del lenguaje. Difieren significativamente en esta variable $(p<0,01)$ del resto de los grupos con TEL (excepto la agnosia verbal y el trastorno léxicosintáctico). La comprensión tiende a ser literal o puede limitarse a frases sencillas y concretas. La expresión verbal es muy fluida, con frases sintácticamente correctas; pero hay que señalar que, una vez evaluada estas capacidades mediante pruebas estructuradas, no es tan adecuada como aparentemente manifiestan. Suelen utilizar frases sin conocer bien el significado, emiten expresiones repetitivas y el discurso puede resultar mal cohesionado. Les cuesta narrar un relato, explicar de manera secuencial una experiencia vivida o expresar una idea de forma coherente. Varios sujetos manifiestan una capacidad de denominación baja; sin embargo, en la bibliografía se señala que, por lo general, el vocabulario suele ser amplio e incluso sofisticado [41,87]. Todas estas dificultades señaladas influyen en la adaptación a la situación comunicativa, por lo que presentan desajustes pragmáticos. Este subtipo comparte características psicolingüísticas con el grupo de Rapin y Allen con 'déficit semántico-pragmático'.

En nuestra casuística no hemos encontrado un déficit específico del contenido del lenguaje; es decir, un trastorno semántico puro. Quizá esta afectación lingüística se da en niños con retraso mental de grado ligero y, como no se incluyó a niños con déficit intelectual, no se contempla en este estudio.

\section{Frecuencia por sexo y subtipo de TEL}

En los 86 sujetos de la muestra la proporción por sexos (2,4 niños por cada niña) se corresponde con la establecida en la bibliografía [6].

Los subtipos que surgen del estudio de conglomerados se dan en nuestra casuística con las frecuencias expresadas en la tabla II. Si tomamos los cuatro primeros subtipos, en los que el trastorno afecta a las dimensiones formales del lenguaje $(n=68)$, el trastorno mixto fonológico-sintáctico afecta a casi la mitad de los sujetos, y le sigue en frecuencia el trastorno fonológico. La frecuencia relativamente elevada del trastorno masivo del lenguaje con agnosia verbal (11\%) puede deberse al reclutamiento de nuestros pacientes en un centro terciario de referencia. La afectación prominente de las dimensiones funcionales del lenguaje -en concreto la pragmática- caracteriza a un $21 \%$ del total de la serie, que se divide en dos subtipos según esté también afectada la semántica (13\%) o no (8\%).

\section{DISCUSIÓN}

Consideramos la denominación TEL la más adecuada para definir el trastorno disfásico porque alude a la especificidad del trastorno y a que éste se produce durante el desarrollo del lenguaje; es decir, matiza que las dificultades lingüísticas no se explican mediante otra u otras patologías y descarta los trastornos del lenguaje adquiridos tras un período de desarrollo normal.

En la identificación de niños con TEL, los criterios de discrepancia cognitiva han desplazado a los de discrepancia cronológica. Así, se pueden evitar falsos positivos (niños cuyas dificultades psicolingüísticas se explican por un retraso mental) y falsos negativos (niños con retraso mental que tienen unas ca- racterísticas lingüísticas inferiores a las esperadas según su edad mental). Sin embargo, por rigor, en este estudio se han utilizado los criterios de exclusión.

En la práctica clínica, para distinguir las formas en que se pueden manifestar los problemas lingüísticos del TEL, consideramos conveniente realizar una evaluación neuropsicolingüística completa del niño que incluya:

- Entrevista semiestructurada con los padres en la que se precisen los hitos del desarrollo psicomotor y la conducta de su hijo.

- Exploración neurológica.

- Evaluación cualitativa del niño mediante un rato de juego con imágenes u objetos.

- Evaluación cuantitativa del rendimiento en las distintas dimensiones del lenguaje mediante pruebas estructuradas, como las utilizadas en este estudio (indicadas en el apartado de 'evaluación psicolingüística').

- Valoración de la información aportada por los padres (el CCC se perfila como un buen instrumento para sistematizar su información en la consulta) y por los distintos profesionales que trabajan con el niño (boletín de notas del maestro, informe del logopeda, psicopedagogo u orientador).

A partir de los resultados de nuestra muestra hispanohablante clasificamos las diferentes categorías diagnósticas del TEL de acuerdo con las capacidades psicolingüísticas que están afectadas de manera predominante:

- Déficit mixto de la vertiente receptiva y expresiva: agnosia verbal auditiva, trastorno fonológico-sintáctico y trastorno léxico-sintáctico.

- Déficit de la vertiente expresiva: trastorno fonológico.

- Déficit de las funciones psicolingüísticas: trastorno semántico-pragmático y trastorno pragmático.

En la tabla III se esquematizan, a modo de resumen, las variables psicolingüísticas que están afectadas en cada una de las variantes del TEL.

En nuestra clasificación no aparece reflejado como subtipo específico la dispraxia verbal. Consideramos que debe incluirse dentro del trastorno fonológico-sintáctico porque los niños afectos de esta patología presentan -además de una alteración en la fonología expresiva- un déficit en la comprensión y expresión de la sintaxis, aspecto al que se ha prestado atención escasa en la bibliografía [41,82,83].

Los dos trastornos con afectación del uso pragmático del lenguaje señalados en nuestra casuística son un fenotipo psicolingüístico peculiar $[58,88]$, que podemos diferenciarlo del síndrome de Asperger porque las habilidades sociales y psicomotrices son apropiadas y no muestran una restricción del campo de intereses. Sin embargo, las relaciones sociales con el grupo de iguales suelen ser pobres, como consecuencia del uso peculiar que le dan al lenguaje, y no es poco frecuente que sean víctimas de discriminación, de un trato social injusto o de acoso escolar [59].

En cada uno de los seis grupos del TEL están afectadas unas habilidades lingüísticas de forma predominante, como se ha señalado anteriormente; pero, al analizar cada una de las dimensiones del lenguaje en todos los sujetos de la muestra, hemos observado en muchos casos que algunas otras capacidades lingüísticas se ven afectadas, como consecuencia del trastorno nuclear que presentan. Por ejemplo, todos los niños evaluados 
Tabla III. Propuesta de clasificación: subtipos clínicos y variables psicolingüísticas afectadas.

\begin{tabular}{|c|c|c|c|c|c|c|c|c|}
\hline & \multicolumn{3}{|c|}{ Recepción } & \multicolumn{3}{|c|}{ Expresión } & \multicolumn{2}{|c|}{ Uso funcional } \\
\hline & Gnosia & Léxico & Sintaxis & Sintaxis & Vocabulario & Fonología & Semántica & Pragmática \\
\hline Agnosia verbal auditiva & ++ & + & + & + & + & + & & \\
\hline Trastorno fonológico-sintáctico & & & + & ++ & + & ++ & & \\
\hline Trastorno léxico-sintáctico & & + & + & ++ & ++ & & + & \\
\hline Trastorno fonológico & & & & & & ++ & & \\
\hline Trastorno semántico-pragmático & & & & & & & ++ & ++ \\
\hline Trastorno pragmático & & & & & & & & ++ \\
\hline
\end{tabular}

-excepto los que pertenecen a los grupos con trastorno fonológico y trastorno pragmático- presentan dificultades de comprensión; éstas se acentúan en el trastorno fonológico-sintáctico y, sobre todo, en la agnosia verbal auditiva. En el grupo léxicosintáctico este bajo rendimiento se explica por la pobreza léxica, y en el grupo semántico-pragmático por los problemas para comprender el significado implícito, que conllevan una interpretación de los mensajes verbales de una forma más o menos literal.

Esto nos indica que las distintas dimensiones del lenguaje no funcionan de manera independiente sino que están íntima- mente relacionadas. Así, los recientes trabajos de genética molecular referidos a la familia $\mathrm{KE}[89,90]$ han mostrado que, con una misma mutación del gen FOXP2, los diferentes componentes de la extensa familia mostraban distintos perfiles de afectación lingüística. Por lo tanto, es necesario reconsiderar los postulados modularistas y adoptar una explicación más integrada de los distintos componentes del lenguaje $[2,38,91]$.

Hay que tener en cuenta, como señalábamos en un trabajo anterior [92], que un mismo sujeto con TEL puede evolucionar y mostrar afectación e indemnidad selectivas de distintos aspectos -formales o funcionales- a lo largo del desarrollo.

\section{BIBLIOGRAFÍA}

1. Bishop D. The underlying nature of specific language impairment. J Child Psychol Psychiatry 1992; 33: 3-66.

2. Leonard L. Children with specific language impairment. 4 ed. Boston: MIT Press; 2002.

3. Chevrie-Muller C. Trastornos específicos del desarrollo del lenguaje. In Narbona J, Chevrie-Muller C, eds. El lenguaje del niño. 2 ed. Barcelona: Masson; 2001. p. 255-82.

4. Narbona J. Trastornos del lenguaje y trastornos del espectro autista en el niño. In: Neurología pediátrica. Madrid: Ergon; 2000.

5. Castaño J. Formas clínicas de las disfasias infantiles. Rev Neurol 2002; 34 (Supl 1): S107-9.

6. Tallal P, Miller S, Bedi G, Byma G, Wang X, Nagarajan S. Temporal processing deficits of language-impaired children ameliorated by training. Science 1996; 271: 77-81.

7. Claustre M. El papel de la comprensión en el trastorno específico de lenguaje durante una conversación dirigida. Revista de Logopedia y Fonoaudiología 1997; 17: 93-102.

8. Miller C, Kail R, Leonard L. Speed of processing in children with specific language impairment. J Speech Lang Hear Res 2001; 44: 416-33.

9. Windsor J, Hwang M. Testing the generalized slowing hypothesis in specific language impairment. J Speech Lang Hear Res 1999; 42: 1205-18.

10. Weismer S, Evans J, Hesketh L. An examination of verbal working memory capacity in children with specific language impairment. J Speech Lang Hear Res 1999; 42: 1249-60.

11. Donlan C, Masters J. Correlates of social development in children with communication disorders: the concurrent predictive value of verbal short-term memory span. Int J Lang Commun Disord 2000; 35: 211-26.

12. Montgomery J. Verbal working memory and sentence comprehension in children with specific language impairment. J Speech Lang Hear Res 2000; 43: 293-308.

13. Gallego C, Revilla P, Schüller M. Recuerdo de material verbal en niños con disfasia funcional. Cognitiva 2000; 12: 37-61.

14. Briscoe J, Bishop D, Norbury C. Phonological processing, language, and literacy: a comparison of children with mild to moderate sensorineural hearing loss and those with specific language. J Child Psychol Psychiatry 2001; 42: 329-40.

15. Evans J, Alibali M, McNeil N. Divergence of verbal expression and embodied knowledge: evidence from speech and gesture in children with language impairment. Lang Cogn Process 2001; 16: 309-31.
16. Bishop D, North T, Donlan C. Nonword repetition as a behavioural marker for inherited language impairment: evidence from a twin study. J Child Psychol Psychiatry 1996; 36: 1-13.

17. Dolloghan C, Campbell T. Nonword repetition and child language impairment. J Speech Lang Hear Res 1998; 41: 1136-46.

18. Conti-Ramsden G, Botting N, Faragher B. Psycholinguistic markers for specific language impairment (SLI). J Child Psychol Psychiatry 2001; 42: 741-8.

19. Martínez L, Bruna A, Guzmán M, Herrera C, Valle J, Vázquez M. El efecto de recencia y el efecto de metría en la memoria de trabajo fonológica en niños preescolares con TEL moderado. Revista Chilena de Fonoaudiología 2001; 2: 19-30.

20. Martínez L, Bruna A, Guzmán M, Herrera C, Valle J, Vázquez M. Alteraciones en las representaciones fonológicas de la memoria de trabajo en niños preescolares con trastorno específico del lenguaje. Revista de Logopedia y Fonoaudiología 2002; 22: 181-9.

21. Conti-Ramsden G. Processing and linguistic markers in young children with specific language impairment. J Speech Lang Hear Res 2003; 46: 1029-37.

22. Conti-Ramsden G, Hesketh A. Risk markers for SLI: a study of young language-learning children. Int J Lang Commun Disord 2003; 38: 251-63.

23. Aguado G, Cuetos-Vega F, Domezáin M, Pascual B. Repetición de pseudopalabras en niños españoles con trastorno específico del lenguaje: marcador psicolingüístico. Rev Neurol 2006; 43 (Supl 1): S201-8.

24. Silva P, McGee R, Williams S. Developmental language delay from three to seven years and its significance for low intelligence and reading difficulties at age seven. Dev Med Child Neurol 1983; 25: 783-93.

25. Brinton B, Fujiki M, McKee L. Negotiation skills of children with specific language impairment. J Speech Lang Hear Res 1998; 41: 927-40.

26. Cohen N, Banwick M, Horodezky N, Vallance D. Language achievement and cognitive processing in psychiatrically disturbed children with previously identified and unsuspected language impairments. J Child Psychol Psychiatry 1998; 39: 865-77.

27. McArthur G, Hogben J, Edwards V, Heath S, Mengler E. On the 'specifics' of specific reading disability and specific language impairment. J Child Psychol Psychiatry 2000; 41: 869-74.

28. Snowling M, Bishop D, Stothard S. Is preschool language impairment a risk factor for dyslexia in adolescence? J Child Psychol Psychiatry 2000; 41: 587-600. 
29. Catts HW, Fey ME, Zhang X, Tomblin JB. Estimating the risk of future reading difficulties in kindergarten children: a research-based model and its clinical implementation. Lang Speech Hear Serv Sch 2001; 32: $38-50$.

30. Nation K, Marshall C, Snowling M. Phonological and semantic contributions to children's picture naming skill: evidence from children with developmental reading disorders. Lang Cogn Process 2001; 16: 241-59.

31. Bishop D, North T, Donlan C. Genetic basis of specific language impairment: evidence from a twin study. Dev Med Child Neurol 1995; 37: $56-71$.

32. SLI Consortium. A genomewide scan identifies two novel loci involved in specific language impairment. Am J Hum Genet 2002; 70: 384-98.

33. Barlett CW, Flax JF, Logue MW. A major susceptibility locus for specific language impairment is located on 13q21. Am J Hum Genet 2002; 71: 45-55.

34. O'Bryen EK, Zhang X, Nishimura C, Tomblin JB, Murray JC. Association of specific language impairment to the region of 7q31. Am J Hum Genet 2003; 72: 1536-43.

35. Bishop D. Uncommon understanding. Development and disorders of language comprehension in children. Hove, UK: Psychology Press; 1997.

36. Aram DM. Comments on specific language impairment as a clinical category. Lang Speech Hear Serv Sch 1991; 22: 84-7.

37. Aguado G. Trastorno específico del lenguaje: diversidad y formas clínicas. Revista Chilena de Fonoaudiología 2002; 3: 48-74.

38. Narbona J, Patiño A. Estudios genéticos de los trastornos de la comunicación. Rev Neurol 2002; 35: 32-6.

39. Rapin I, Allen D. Developmental dysphasia and autism in preschool children: characteristics and subtypes. In Martin J, Martin P, Fletcher P, Grunwell P, Hall D, eds. Proceedings of the First International Symposium on Specific Speech and Language Disorders in Children. London: Association For All Speech Impaired Children (AFASIC); 1987. p. 20-35.

40. Rapin I, Allen D. Syndromes in developmental dysphasia and adult aphasia. In Plum F, ed. Language, communication and the brain. New York: Raven Press; 1988. p. 57-75.

41. Tuchman RF, Rapin J, Shinnar S. Autistic and dysphasic children I: clinical characteristics. Pediatrics 1991; 88: 1211-8.

42. Rapin I. Developmental language disorders: a clinical update. J Child Psychol Psychiatry 1996; 37: 643-56.

43. Aram DM, Nation J. Patterns of language behavior in children with developmental language disorders. J Speech Hear Res 1975; 18: 229-41.

44. Conti-Ramsden G, Crutchley A, Botting N. The extent to which psychometric tests differentiate subgroups of children with specific language impairment. J Speech Lang Hear Res 1997; 40: 765-77.

45. Conti-Ramsden G, Botting N. Classification of children with specific language impairment: longitudinal considerations. J Speech Lang Hear Res 1999; 42: 1195-204.

46. Rivière A. Tratamiento y definición del espectro autista I. Relaciones sociales y comunicación. In Rivière A, Martos J, eds. El tratamiento del autismo: nuevas perspectivas. Madrid: IMSERSO-APNA; 1997. p. 61-106.

47. Fattal-Valevski A, Kramer U, Leitner Y, Nevo Y, Greenstein Y, Harel S. Characterization and comparison of autistic subgroups: 10 years' experience with autistic children. Dev Med Child Neurol 1999; 41: 21-5.

48. Allen DA, Rapin I. Autistic children are also dysphasic. In Naruse H, Ornitz E, eds. Neurobiology of infantile autism. Amsterdam: Excerpta Medica; 1992. p. 73-80.

49. Belinchón M, Rivière A, Igoa JM. Psicología del lenguaje. Investigación y teoría. Madrid: Trotta; 1992.

50. Rapin I. Trastornos de la comunicación en el autismo infantil. In Narbona J, Chevrie-Muller C, eds. El lenguaje del niño. Barcelona: Masson; 2001. p. 365-82.

51. Wing L. The autistic spectrum. Lancet 1997; 350: 1761-6.

52. Bishop D, Frazier-Norbury C. Exploring the borderlands of autistic disorder and specific language impairment: a study using standardised diagnostic. J Child Psychol Psychiatr 2002; 43: 917-29.

53. Bishop DV. Autism, Asperger's syndrome and semantic-pragmatic disorder: where are the boundaries? Br J Disord Commun 1989; 24: 107-21.

54. Shields J, Varley R, Broks P, Simpson A. Social cognition in developmental language disorders and high-level autism. Dev Med Child Neurol 1996; 38: 487-95.

55. American Psychiatric Association. Manual diagnóstico y estadístico de los trastornos mentales: DSM-IV TR. Barcelona: Masson; 2003.

56. Organización Mundial de la Salud. CIE-10. Clasificación de los trastornos mentales y del comportamiento. Madrid: Editorial Panamericana; 2000.

57. Gagnon L, Mottron L, Joanette Y. Questioning the validity of the semantic-pragmatic syndrome diagnosis. Autism 1997; 1: 37-55.

58. Bishop D. Pragmatic language immpairment: a correlate of SLI, a distinct subgroup, or part of the autistic continuum? In Bishop D, Leonard
L, eds. Speech and language impairments in children. Causes, characteristics, intervention and outcome. Hove, UK: Psychology Press; 2000. p. 99-113.

59. Conti-Ramsden G, Botting N. Social difficulties and victimization in children with SLI at 11 years of age. J Speech Lang Hear Res 2004; 47: 145-61.

60. Liiva CA, Cleave PL. Roles of initiation and responsiveness in access and participation for children with specific language impairment. J Speech Lang Hear Res 2005; 48: 868-83.

61. Marton K, Abramoff B, Rosenzweig S. Social cognition and language in children with specific language impairment (SLI). J Commun Disord 2005; 38: 143-62.

62. McCabe PC. Social and behavioral correlates of preschoolers with specific language impairment. Psychol Sch 2005; 42: 373-87.

63. Juárez A, Monfort M. Estimulación del lenguaje oral. Madrid: Enthaediciones; 2001.

64. Monfort M, Juárez A. Niños con trastornos pragmáticos del lenguaje y de la comunicación. Madrid: Enthaediciones; 2004.

65. Adams, C. Social communication intervention for school-age children: rationale and description. Semin Speech Lang 2005; 26: 181-8.

66. Fresneda MD, Mendoza E. Trastorno específico del lenguaje: concepto, clasificaciones y criterios de identificación. Rev Neurol 2005; 41 (Supl 1): S51-6.

67. Leiter, R. Leiter Inteligence Perfomance Scale: LIPS. Madrid: MEPSA; 1948.

68. Roid G, Miller L. Leiter International Performance Scale. Windsor: Nelson; 1996

69. Wechsler D. Escala de inteligencia para niños, revisada: WISC-R. Madrid: TEA; 1993

70. De la Osa N, Doménech E, Narbona J, Chevrie-Muller C. Batería de exploración del lenguaje para preescolares: BELP-P. Revista de Logopedia y Fonoaudiología 1994; 2: 108-16.

71. Kirk S, Kirk W. Test Illinois de aptitudes psicolingüísticas (ITPA). Adaptación española. Madrid: TEA; 1986.

72. Dunn L, Padilla E, Lugo D, Dunn L. Test de vocabulario en imágenes Peabody: TVIP. Adaptación hispanoamericana. Madrid: TEA; 1986.

73. Aguado G. El desarrollo de la morfosintaxis en el niño: TSA. Manual de evaluación. Madrid: CEPE; 1989.

74. Spreen O, Benton A. Neurosensory center comprehensive examination for aphasia. Manual of instruction. Victoria: University of Victoria, Neuropsychology Laboratory; 1969.

75. Mendilaharsu C, Gaudiano J, Flores de Rodríguez M, Luque S, López de Cayaffa C, Benavídez C, et al. Batería de pruebas para el estudio del lenguaje del niño, de acuerdo al modelo de Spreen y Benton. In Mendilaharsu C, ed. Estudios neuropsicológicos. Vol. III. Montevideo: Delta; 1981. p. 137-63.

76. Monfort M, Juárez A. Registro fonológico inducido: RFI. Madrid: CEPE; 1989.

77. Goodglass H, Kaplan E. Test de vocabulario de Boston: TVB. Madrid: Panamericana; 1996.

78. McCarthy D. Escalas de aptitudes y psicomotricidad para niños: MSCA. Madrid: TEA; 1986

79. Reynolds C, Bigler E. Test de memoria y aprendizaje (TOMAL). Adaptación española. Madrid: TEA; 2001

80. Bishop D. Development of the Children's Communication Checklist (CCC): a method for assessing qualitative aspects of communicative impairment in children. J Child Psychol Psychiatry 1998; 39: 879-91.

81. Bishop D, Baird G. Parent and teacher report of pragmatic aspects of communication: use of the Children's Communication Checklist in a clinical setting. Dev Med Child Neurol 2001; 43: 809-18.

82. Hall P, Jordan L, Robin D. Developmental apraxia of speech. Austin, TX: Pro-Ed; 1993.

83. Shriberg LD, Aram DM, Kwiatkowski J. Developmental apraxia of speech: I. Descriptive and theoretical perspectives. J Speech Lang Hear Res 1997; 40: 273-85.

84. Gleitman L. The structural sources of verb meaning. Language Acquisition 1990; 1: 3-55

85. Van der Lely H. Canonical linking rules: forward versus reverse linking in normally developing and specifically language-impaired children. Cognition 1994; 51: 29-72.

86. Fisher C. Structural limits on verb mapping: the role of abstract structure in 2.5-year-olds' interpretations of novel verbs. Dev Sci 2002; 5: 55-64.

87. Firth C, Venkatesh K. Semantic-pragmatic language disorder. Oxon: Winslow; 1999.

88. Rapin I, Allen D. Developmental language disorders: nosologic considerations. In Kirk V, ed. Neuropsychology of language, reading and spelling. New York: Academic Press; 1983. p. 155-84

89. Vargha-Khadem F, Watkins K, Alcock K, Fletcher P, Passingham R. 
Praxic and nonverbal cognitive deficits in a large family with a genetically transmitted speech and language disorder. Proc Natl Acad Sci USA 1995; 92: 930-3.

90. Lai C, Fisher SE, Hurst JA, Vargha-Khadem F, Monaco AP. A forkhead-domain gene is mutated in a severe speech and language disorder. Nature 2001; 413: 519-23.

\section{SUBTIPOS DE TRASTORNO ESPECÍFICO DEL DESARROLLO DEL LENGUAJE: PERFILES CLINICOS EN UNA MUESTRA HISPANOHABLANTE}

Resumen. Introducción. Los subtipos internacionalmente admitidos en el continuo de los trastornos específicos del desarrollo del lenguaje (TEL) derivan del análisis de niños angloparlantes, pero no se han contrastado suficientemente hasta ahora en una población con el castellano como idioma de base. Objetivo. Se trata de obtener una clasificación derivada de la fenomenología clínica de pacientes hispanohablantes. Pacientes y métodos. 86 pacientes correlativos, de 4 a 9 años, que cumplen criterios clínicos de TEL, con un cociente intelectual no verbal entre 72 y 114 (media: 93). A todos se les aplicó un protocolo de entrevista y de pruebas para medir sus capacidades lingüisticas en los aspectos formales y funcionales. Los datos se han sometido a análisis de conglomerados. Resultados. Del total de la serie, un $24 \%$ presenta un trastorno limitado a la programación fonológica expresiva. En otro $55 \%$ concurren trastornos mixtos en las vertientes de comprensión y de expresión, que van desde la afectación masiva derivada de una agnosia verbal congénita (11\%) a trastornos menos graves que combinan dificultad en la sintaxis receptiva y expresiva con trastorno de la fonología (36\%) o de acceso al léxico (8\%). Por otra parte, el $21 \%$ de la serie se caracteriza por dificultades en la pragmática del lenguaje, pese a que tanto el dominio formal de las reglas lingüísticas como la conducta extraverbal estén en límites aceptables. Conclusiones. Nuestro estudio confirma la adecuación, en niños hispanohablantes, de los subtipos de TEL actualmente admitidos en la bibliografía internacional, con vistas al fenotipado y a la planificación de estrategias de intervención. [REV NEUROL 2006; 43 (Supl 1): S193-200]

Palabras clave. Agnosia auditiva verbal. Disfasia. Niños hispanohablantes. Pragmática. Trastorno específico del lenguaje. Trastorno fonológico. Trastorno fonológico-sintáctico. Trastorno léxicosintáctico.
91. Joanisse M, Seidenberg MS. Phonology and syntax in specific language impairment: evidence from a connectionist model. Brain Lang 2003; 86: 40-56

92. Crespo-Eguílaz N, Narbona J. Perfiles clínicos evolutivos y transiciones en el espectro del trastorno específico del desarrollo del lenguaje. Rev Neurol 2003; 36 (Supl 1): S29-35.

\section{SUBTIPOS DE PERTURBAÇ̃̃O ESPECÍFICA DO DESENVOLVIMENTO DA LINGUAGEM: PERFIS CLÍNICOS NUMA AMOSTRA DE FALANTES DE LÍNGUA ESPANHOLA}

Resumo. Introdução. Os subtipos internacionalmente aceites como um contínuo das perturbações específicas do desenvolvimento da linguagem (PEL) derivam da análise de crianças falantes anglosaxónicas, não existindo porém, estudos com uma população que tenha o castelhano como língua de origem. Objectivo. Trata-se de obter uma classificação derivada da fenomenologia clínica de doentes falantes de língua espanhola. Doentes e métodos. Foram estudados 86 doentes correlativos, de 4 a 9 anos, que cumpram critérios clínicos de PEL, com um quociente de inteligência não verbal entre 72 e 114 (média: 93). Foi aplicado um protocolo de entrevista e de testes para medir os aspectos formais e funcionais das suas capacidades linguísticas. Os dados foram submetidos a uma análise de cluster. Resultados. Do total da série, $24 \%$ apresentam uma perturbação limitada à programação fonológica expressiva. Outros $55 \%$ manifestam perturbações mistas nas vertentes de compreensão e de expressão, que vão desde a afecção maciça derivada de uma agnosia verbal congénita $(11 \%)$ a perturbações menos graves que combinam a dificuldade na sintaxe receptiva e expressiva com a perturbação da fonologia (36\%) ou de acesso ao léxico (8\%). Por outro lado, $21 \%$ da série caracterizam-se por dificuldades na pragmática da linguagem. No entanto, quer o domínio formal das regras linguísticas como da conduta extraverbal permanecem dentro de limites aceitáveis. Conclusões. O nosso estudo confirma a adequação, em crianças falantes de língua espanhola, dos subtipos de PEL actualmente aceites na bibliografia internacional, com o objectivo de determinar o fenótipo e planificar estratégias de intervenção. [REV NEUROL 2006; 43 (Supl 1): S193-200]

Palavras chave. Agnosia auditiva verbal. Crianças falantes de língua espanhola. Disfasia. Perturbação específica da linguagem. Perturbação fonológica. Perturbação fonológico-sintáctica. Perturbação léxico-sintáctica. Pragmática. 